Revista peruana de biología 26(2): 235 - 242 (2019) doi: http://dx.doi.org/10.15381/rpb.v26i2.16380 ISSN-L 1561-0837; eISSN: 1727-9933

Universidad Nacional Mayor de San Marcos

\section{Estado de conservación de Myrcianthes ferreyrae un árbol endémico de las lomas costeras del sur del Perú}

\section{TRABAJOS ORIGINALES}

$\begin{array}{ll}\text { Presentado: } & 19 / 01 / 2019 \\ \text { Aceptado: } & 02 / 03 / 2019 \\ \text { Publicado online: } & 06 / 07 / 2019\end{array}$

Correspondencia:

*Autor para correspondencia:

Fiorella N. Gonzales: nasharellas@yahoo.es

Francisco Villasante Benavides: fvillabe@gmail.com

Otros datos de los autores / biografía:

ORCID Fiorella N. Gonzales: 0000-0001-5240-2464

ORCID Francisco Villasante: 0000-0002-6577-3122

Citación:

Gonzales Guillén F.N. \& F. Villasante Benavides. 2019. State of conservation de Myrcianthes ferreyrae an endemic tree of the coastal hills of southern Peru. Revista peruana de biología 26(2): 235 - 242 (Julio 2019). doi: http://dx.doi.org/10.15381/rpb. v26i2.16380

Palabras clave: Myrtaceae; extinción; conservación; lomas costeras; regeneración; desierto peruano. Keywords: Myrtaceae; extinction; conservation; coastal hills; regeneration; Peruvian desert.

\section{State of conservation de Myrcianthes ferreyrae an endemic tree of the coastal hills of southern Peru}

\section{Fiorella N. Gonzales Guillén*1,2 y Francisco Villasante Benavides ${ }^{3}$}

1. Museo de Historia Natural de la Universidad Nacional de San Agustín de Arequipa, Av. Alcides Carrión s/n, Arequipa, Perú.

2. Departamento de Ecología, Pontificia Universidad Católica de Chile, Avda. Libertador Bernardo O'Higgins 340, Santiago, Chile.

3. Sección de Ecología y Conservación, Departamento Académico de Biología, Facultad de Ciencias Biológicas y Agropecuarias, Universidad Nacional de San Agustín de Arequipa, Av. Alcides Carrión s/n, Arequipa. Perú.

\section{Resumen}

Myrcianthes ferreyrae (McVaugh) McVaugh, el "arrayan" es un árbol endémico y amenazado de las lomas costeras del sur de Perú. El presente trabajo evaluó su estado de conservación entre marzo 2011 a agosto 2012. Se realizó el conteo de todos los individuos en casi todo su rango de distribución conocido (lomas de Atiquipa, Taymara y Chala viejo en la Provincia de Caraveli, Arequipa); se estableció un total de 9 parcelas de 0.25 ha, tres en cada localidad y en cada una se tomaron datos estructurales de todos los árboles con un diámetro un basal mayor a $10 \mathrm{~cm}$; además, se evaluaron parámetros de mortalidad, regeneración natural y la presencia de disturbios. El arrayan tiene una población de 586 individuos, presenta una distribución diamétrica en forma de campana, la categoría de mortalidad más común es la tala de individuos; el porcentaje de regeneración natural es $33.12 \%$, todos pertenecientes a las lomas de Atiquipa. Se concluye que poblacion de $M$. ferreyrae esta fuertemente amenazada, conformada por individuos adultos y con poca regeneración natural. En el manejo de esta especie debe considerarse su fragilidad y los problemas que tiene para su regeneración.

\section{Abstract}

Myrcianthes ferreyrae (McVaugh) McVaugh, "arrayan" is an endemic and threatened tree of the "lomas" formations of south Peru. We evaluated their conservation status between March 2011 to August 2012; for which, all individuals were counted in its distributional range known (Atiquipa, Taymara and Chala Viejo hills in the Province of Caraveli, Arequipa). We established 9 plots of 0.25 hectares, three in each locality; in each plot we obtained data of all trees greater than $10 \mathrm{~cm}$ basal diameter, plus mortality parameters, its natural regeneration and presence of disturbances. Myrcianthes ferreyrae has a population of 586 individuals, its diameter distribution shows a bell form, the most common category mortality was logging; the natural regeneration was $33.12 \%$, all belonging to the Atiquipa hills. We conclude that $M$. ferreyrae is strongly threatened, formed by adult individuals and with little natural regeneration. In management of $M$. ferreyrae should be considered its fragility and the problems it has for its regeneration. 


\section{Introducción}

Las lomas costeras son ecosistemas exclusivos del desierto costero del Pacífico sudamericano y se caracterizan por presentar una vegetación altamente estacional sustentada por las neblinas invernales que se condensan en las laderas de los cerros costeros (Ferreyra 1983). Estas formaciones vegetales se presentan por el norte hasta Trujillo ( $8^{\circ} \mathrm{S}$, Perú) y por el sur hasta Coquimbo $\left(30^{\circ} \mathrm{S}\right.$, Chile) (Ferreyra 1986). Brack y Mendiola (2012) clasifican las lomas según su vegetación en: lomas de árboles, lomas de arbustos y hierbas, lomas de suculentas y lomas de plantas xerofitas y semixerofitas. Las lomas arbóreas paulatinamente han disminuido debido a la deforestación (Ferreyra 1983) y al sobrepastoreo (Pefaur 1982).

Existen numerosas especies endémicas en las formaciones de lomas, una de ellas el "arrayan" Myrcianthes ferreyrae McVaugh (McVaugh), especie arbórea perennifolia siempre verde (Brako y Zarucchi 1993), perteneciente a la familia Myrtaceae. Su distribución está limitada a algunas lomas en la provincia de Caraveli (Arequipa), esta especie es dominante en las lomas de Taimara, donde presenta baja densidad poblacional y no se observa regeneración natural (Rundel et al. 1991). Myrcianthes ferreyrae es categorizada en "Peligro crítico" por la legislación peruana (DS N43-2006-AG) y como "Críticamente Amenazada" por la Unión Internacional para la Conservación de la Naturaleza (Gonzales 2014). Los únicos trabajos que se han realizado en esta especie están referidos a la cantidad de riego que necesitan sus plantones reforestados (Ramírez et al. 2011, Mancilla 2010) y su fenología en las lomas de Atiquipa (Álvarez 2014).
El presente trabajo tiene como objetivo documentar la distribución, abundancia y estructura poblacional de M. ferreyrae en las lomas de Atiquipa, Taimara y Chala Viejo, documentando el estado de conservación y grado de amenaza que afronta esta especie.

\section{Material y métodos}

Área de estudio.- La evaluación se realizó entre marzo 2011 a agosto 2012. El área de estudio corresponde a las lomas de Atiquipa (1546'55.6”S, 74²2'44.9”W), Taymara (154 $\left.47^{\prime} 6.27^{\prime \prime S}, 7^{\circ} 19^{\prime} 47.22^{\prime \prime} \mathrm{W}\right)$ y Chala Viejo $\left.15^{\circ} 45^{\prime} 7.21^{\prime \prime} \mathrm{S}, 7^{\circ} 14^{\prime} 45.54^{\prime \prime} \mathrm{W}\right)$, ubicadas en la parte norte del departamento de Arequipa, provincia de Caravelí (Fig. 1). Se encuentra en la zona de vida desierto per árido templado cálido (ONERN 1976), en la ecorregión del Desierto Costero (Brack 1986) y en el piso climático Termotropical hiperárido con una temperatura media anual de $18.5^{\circ} \mathrm{C}$ y precipitación media anual de $17 \mathrm{~mm}$ (Galán de Mera et al. 2010).

Distribución geográfica, abundancia y densidad.Se utilizó datos de posicionamiento global de los árboles de arrayan provenientes de las lomas de Atiquipa y Taymara (información proporcionada por Aldo Ortega), los cuales fueron confirmados en campo, contándose y georreferenciándose nuevos individuos si fuera el caso, tal como sucedió en las lomas de Chala Viejo. Además, se revisó bibliografía y la colección del herbario del Museo de Historia Natural de la Universidad Mayor de San Mar$\cos$ (USM).

Adicionalmente, se estimaron parámetros de abundancia (mediante el conteo total de individuos en cada
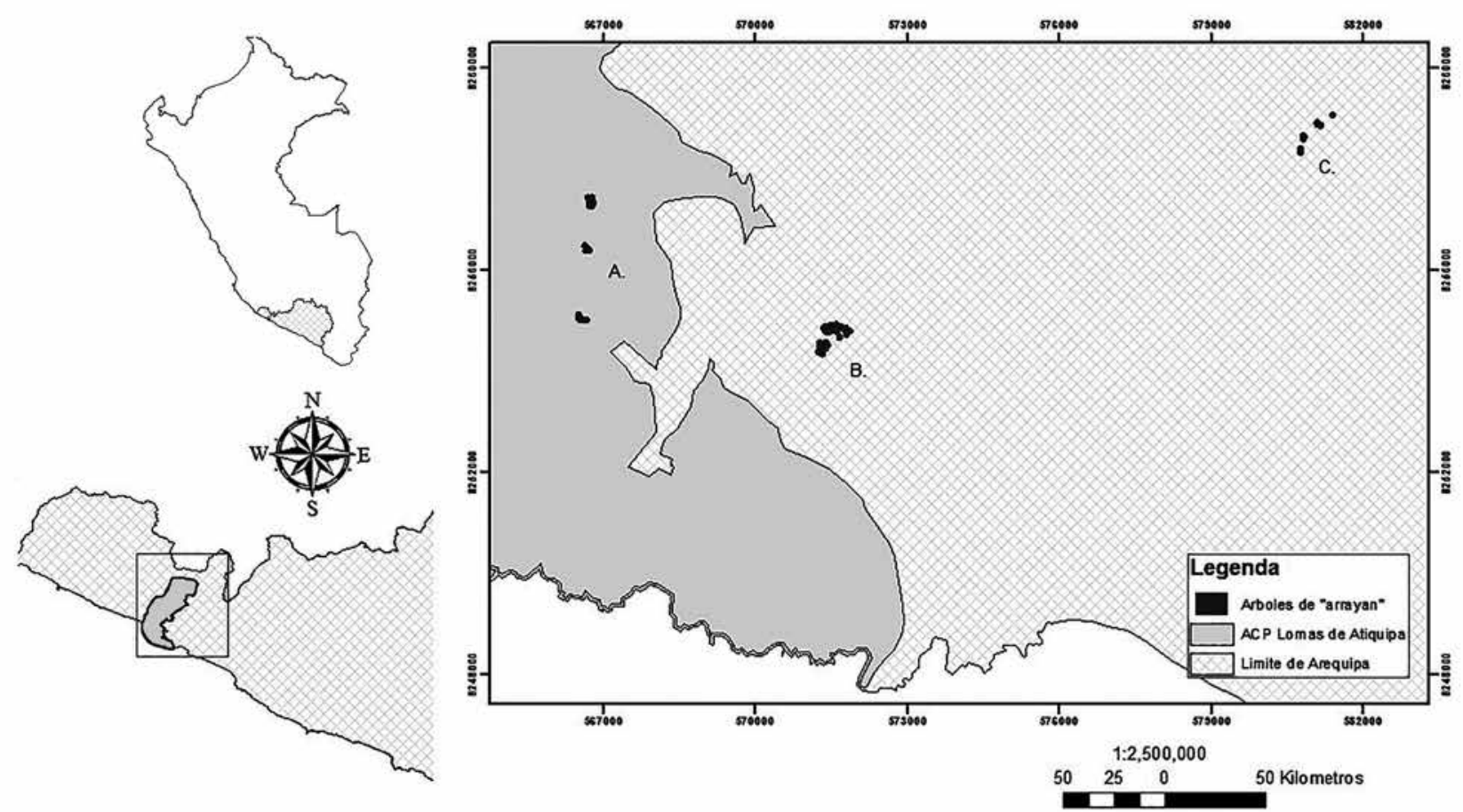

Figura 1. Mapa de las localidades evaluadas de M. ferreyrae, en el presente estudio. A. Lomas de Atiquipa, B. Lomas de Taimara, C. Lomas de Chaparra, en la provincia de Caravelí, departamento de Arequipa. 
localidad evaluada) y densidad (número de individuos por la unidad de superficie (ha) en cada área evaluada).

Estructura poblacional.- Se ubicaron al azar seis parcelas de 0.25 ha $(50 \times 50 \mathrm{~m})$ en un mapa con datos de presencia de "arrayan" en las Lomas de Atiquipa (en las localidades de Conchara, Avalos, Huayahuani) y tres en las Lomas de Taimara. Para las lomas de Chala Viejo, por no contar con árboles previamente georrefenciados se estableció en campo tres parcelas al azar con las características mencionadas anteriormente, en los lugares donde se verificó la presencia de la especie. Todas las parcelas fueron trazadas siguiendo las recomendaciones de Contreras et al. (1999). Las variables medidas fueron altura total, diámetro a la altura del pecho (DAP), regeneración natural y mortalidad. El DAP fue medido en todos los árboles con perímetros del tronco mayores a $10 \mathrm{~cm}$ a $1.30 \mathrm{~m}$ del nivel del suelo. Para casos especiales se empleó lo descrito por Contreras et al. (1999), para obtener el diámetro a la altura del pecho, la medición obtenida se dividió por $\pi$ (3.1416). Estos datos se clasificaron en clases diamétricas: Clase I ( 3 a 9.9 cm), II (10 a $19.9 \mathrm{~cm}$ ), III (20 a 29,9 cm), IV (30 a $39.9 \mathrm{~cm})$, V (40 a $49.9 \mathrm{~cm}$ ), VI (50 a 59,9 cm).

Regeneración natural.- Para la evaluación de la regeneración natural, en cada parcela se contabilizaron los individuos de 0 a $150 \mathrm{~cm}$ de altura categorizándolos en (a) plántulas (semillas germinadas con cotiledones extendidos hasta $0.30 \mathrm{~m}$ de altura), (b) brinzales (altura entre $0.30 \mathrm{~m}$ y $1.5 \mathrm{~m}$ ) siguiendo las consideraciones de Hutchinson (1993) en Hernandez (1999). El porcentaje de regeneracion natural se obtuvo dividiendo el número de rebrotes por el total de adultos por cada localidad.

Mortalidad.- La mortalidad se evaluó de acuerdo a la clasificación propuesta por Melo y Vargas (2003), considerándose árbol muerto en pie cuando el individuo se encontraban en pie sin daños evidentes en su fuste o ramas, tronco partido cuando se encontraba con fragmentos del fuste en pie y otros en el suelo, caído de raíz cuando se encontraba completamente caído en el suelo con las raíces expuestas, cortado cuando había evidencia de tocones y, sin clasificación individuos que no pueden ser categorizados.

Degradación.- La degradación del bosque se evaluó por la presencia de perturbaciones obvias de impacto como tala (presencia de tocones) y ganado (signos de ramoneo, presencia de sendas, de ganado y excrementos) o sin disturbios (Rovere 2002).

Análisis de datos.- El mapa con los registros de la especie se realizó con el programa ArGis 10.2. Los shapefiles utilizados (centros poblados, límites distritales) se obtuvieron a partir de la Carta Geológica Nacional publicada por el Instituto Geográfico Nacional. La estructura poblacional (DAP y Mortalidad) se analizaron con base en histogramas de frecuencia y se graficaron con el programa StatisGraphic 3.2.

\section{Resultados y discusión}

Distribución geográfica y espacial.- El arrayan se encuentra distribuido en las lomas de Atiquipa, Taimara y Chala Viejo, además, en las lomas El Cali (com pers. Carmelo Talavera), lomas de Chaparra (localidad tipo) (McVaugh 1956, Rundel et al. 1991, USM 27843, USM 27846) y Lomas de Acari (USM 27874) todas ubicadas en la Provincia de Caravelí, departamento de Arequipa (Tabla 1), llegando a crecer desde los 500 hasta los 900 $m$ de altitud. Presenta una disposición espacial agregada en quebradas con pendientes pronunciadas. Consideramos que todas las lomas estudiadas (Atiquipa, Taimara y Chala Viejo) se comportan como poblaciones naturales, es decir individuos de la misma especie compartiendo un lugar, que se delimitan por barreras naturales ecológicas o genéticas (Andrewartha \& Birch 1984). En este caso las estamos delimitando por barreras naturales, el desierto costero, que las separa unas de otras. A pesar que se considera que formaban en el pasado un continuo y fueron fragmentadas por el sobrepastoreo y la tala excesiva (Ferreyra 1983, Rundel et al. 1991). Consideramos que la probabilidad de los individuos de emigrar o inmigrar entre cada loma serian eventos limitados o nulos, y que es posible que no haya pasado suficiente tiempo para que se vea una diferenciación genética a nivel poblacional. En las lomas de Atiquipa la especie está distribuida en tres parches (Avalos, Conchara y Huayahuani) con 27, 15 y 24 árboles respectivamente; en Taimara esta forma dos parches con 470 individuos en total, en Chala Viejo se distribuye en un solo parche con 50 individuos (Fig. 1).

Abundancia.- En total se registraron 586 individuos adultos (Tabla 2). En las lomas de Atiquipa y Chala Vie-

Tabla 1.- Localidades donde se encuentra distribuida Myrcianthes ferreyrae. $\left({ }^{*}\right)$ Localidades no muestreadas en este estudio

\begin{tabular}{|c|c|c|c|c|c|}
\hline $\begin{array}{l}\text { Departamento } \\
\text { (provincia) }\end{array}$ & Localidades & Coordenadas UTM(WGS 84) & $\begin{array}{l}\text { Altura } \\
(\mathrm{m})\end{array}$ & $\begin{array}{c}\text { Pendiente } \\
\left({ }^{\circ}\right)\end{array}$ & Registro \\
\hline \multirow{6}{*}{$\begin{array}{l}\text { Arequipa, } \\
\text { Caraveli }\end{array}$} & Lomas de Atiquipa & $15^{\circ} 46^{\prime} 55.6^{\prime \prime} \mathrm{S}, 74^{\circ} 22^{\prime} 44.9^{\prime \prime} \mathrm{W}$ & 740 & 20 & \multirow{5}{*}{$\begin{array}{l}\text { USM 277206, USM } 237263 \\
\text { Mc Vaught (1958), PO Combelles 1958, Ferreyra } \\
\text { 1959, Del Carpio } 1986 \\
\text { Com pers. Carmelo, Este estudio } \\
\text { Mc Vaugh 1956, Rundel et al. 1991, USM 27843, } \\
\text { USM } 27846 \\
\text { Com pers. Carmelo Talavera } 2013\end{array}$} \\
\hline & Lomas de Taimara & $15^{\circ} 47^{\prime} 6.27^{\prime \prime} \mathrm{S}, 74^{\circ} 19^{\prime} 47.22^{\prime \prime} \mathrm{W}$ & 580 & 33 & \\
\hline & Lomas de Chala Viejo & $15^{\circ} 45^{\prime} 7.21^{\prime \prime} \mathrm{S}, 74^{\circ} 14^{\prime} 45.54^{\prime \prime} \mathrm{W}$ & 900 & \multirow[t]{3}{*}{28} & \\
\hline & *Lomas de Chaparra & $15^{\circ} 49^{\prime} 59.9^{\prime \prime} \mathrm{S}, 74^{\circ} 0^{\prime} 21.39^{\prime \prime} \mathrm{W}$ & 650 & & \\
\hline & *Lomas El Cali & $15^{\circ} 50^{\prime} 55.359^{\prime \prime} \mathrm{S}, 74^{\circ} 10^{\prime} 33.48^{\prime \prime} \mathrm{W}$ & 600 & & \\
\hline & *Lomas de Acari & $15^{\circ} 26^{\prime} 18.4^{\prime \prime S}, 74^{\circ} 37^{\prime} 5.09^{\prime \prime} \mathrm{W}$ & 900 & & USM 27847 \\
\hline
\end{tabular}


Tabla 2.- Densidad de los árboles de Myrcianthes ferreyrae para cada localidad evaluada y distancia (km) entre las localidades más cercanas.

\begin{tabular}{lcccc}
\hline Localidades & Área (ha) & $\mathbf{N}^{\circ}$ de individuos & Densidad (ind/ha) & Distancia más cercana entre localidades (km) \\
\hline Atiquipa (Avalos) & 1.02504 & 27 & 26.34 & 1.484438 (Conchara) \\
$\quad$ (Conchara) & 0.42897 & 15 & 34.96 & 1.019616 (Huayahuani) \\
$\quad$ (Huayahuani) & 0.915458 & 24 & 26.22 & 1.019616 (Conchara) \\
Taimara & 9.948737 & 470 & 47.24 & 4.829321 (Avalos) \\
Chala Viejo & 2.441034 & 50 & 20.48 & 9.543417 (Taimara) \\
\hline Total & 14.759239 & 586 & 39.7 & \\
\hline
\end{tabular}

jo esta especie crece asociada principalmente a la tara Caesalpinea spinosa y en las lomas de Taimara es la especie dominante acompañada de pocos individuos de tara. No existen datos de abundancia para las otras localidades.

Densidad.- Myrcianthes ferreyrae presenta una densidad promedio de 39.70 árboles/ha en el total de la superficie evaluada. Taimara presenta la mayor densidad con 47 árboles/ha en un área de casi 10 ha de extensión, la localidad que presenta menor densidad es Chala Viejo con 20.5 árboles/ha en un área de casi 2.5 ha (Tabla 2). CIZA-ORNERN-SENAMHI (1989) reportaron en Taimara una densidad de 86 árboles/ha, mientras que nuestros datos resultaron en 47 árboles/ha, lo que podria reflejar una disminución continua en la población, posiblemente por presión antrópica (tala). Aunque Vizcarra (2004) indica una densidad de 2.698 árboles/ha en Atiquipa, consideramos que este valor esta sesgado porque en ese estudio no se consideró el tipo de distribución agregada que presenta el arrayan en Atiquipa, donde se encuentra limitada a tres parches. No existe información sobre las poblaciones de las otras localidades donde se distribuye la especie (Tabla 1).

Distribución diamétrica.- la distribución diamétrica presenta una forma de campana (Fig. 2); característica de especies con problemas de regeneración (Lamprecht
1990 en Vílchez 2005). Las categorías intermedias II y III fueron las más abundantes. El bajo porcentaje de árboles en la clase diamétrica I, podría estar asociado como se mencionó anteriormente, a problemas de regeneración producidos posiblemente por la herbivoría, debido a la existencia de ganado caprino y vacuno (sobrepastoreo).

La falta de árboles en las clases de mayor diámetro V y VI al parecer es por la tala excesiva, reportada previamente por Ferreyra (1983), Rundel et al. (1991), Canziani (1997) y Suasnabar et al. (2004), o por la muerte de los árboles viejos producto de la estocasticidad (v.g. sequias durante fenómenos ENSO, plagas de insectos). Es importante mencionar el efecto del cambio climático sobre las lomas costeras el cual se encontraría incrementando la aridez, estos efectos ya han sido evidenciados en los ecosistemas de lomas de Chile y Perú (Schulz et al. 2011). Tambien, se conoce que la sequía incrementa la mortalidad de los árboles, causando principalmente falla hidráulica, inanición de carbono y alteraciones en su metabolismo fisiológico (Allen et al. 2010).

Referente a la estructura poblacional por cada localidad (Fig. 3), Atiquipa mostró un patrón descendente, esto es, la mayoría de árboles pertenecían a las categorías menores y con ausencia de árboles grandes. En cambio, en las lomas de Taimara, se observó una distribución en forma de campana caracterizada por la mayor cantidad de árbo-

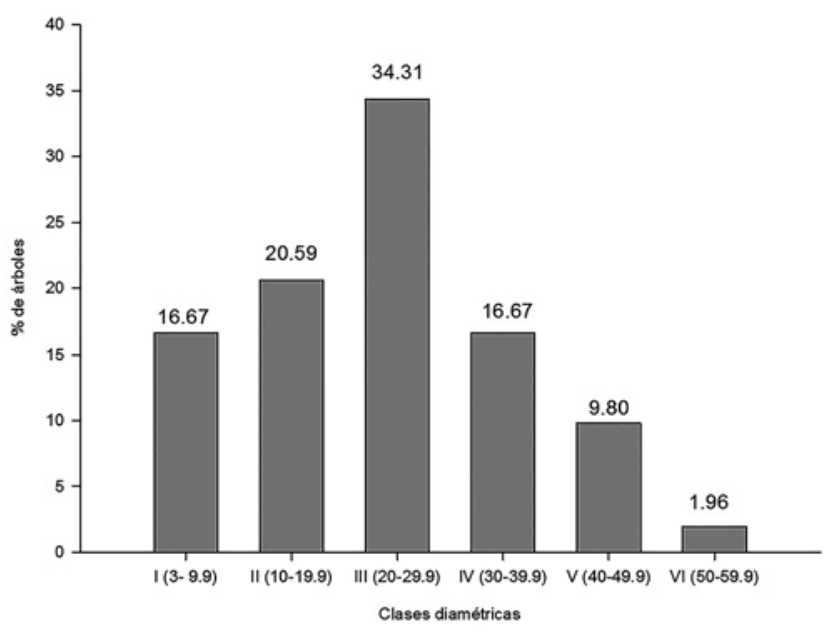

Figura 2.- Distribucion porcentual por clase diametrica de árboles de Myrcianthes ferreyrae en todas las localidades evaluadas.

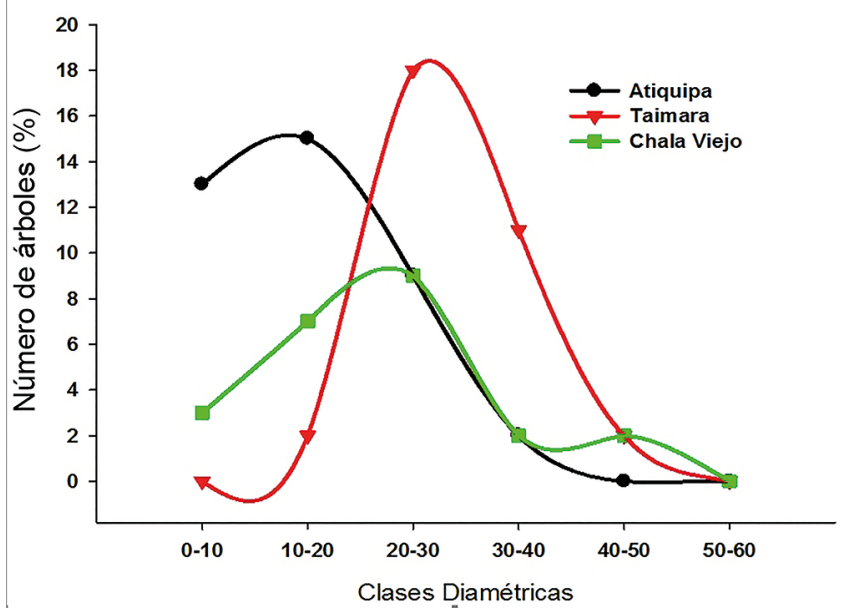

Figura 3.- Numero de árboles de Myrcianthes ferreyrae por clase diamétrica en cada localidad evaluada. 
Tabla 3.- Número de individuos adultos y juveniles (brinzales y plántulas) de Myrcianthes ferreyrae y porcentaje de regeneración por cada localidad, obtenidos en nueve parcelas de 0.25 ha, durante los meses de marzo 2011 a agosto 2012.

\begin{tabular}{lcccc}
\hline Localidad & Adultos & Brinzales & Plántulas & \% de regeneración \\
\hline Atiquipa & 47 & 36 & 18 & 53.46 \\
Taimara & 44 & 0 & 0 & 0 \\
Chala Viejo & 18 & 0 & 0 & 0 \\
\hline Total & 109 & 36 & 18 & 33.12 \\
\hline
\end{tabular}

les en las categorías intermedias y pocos en las extremas. Finalmente, en las lomas de Chala Viejo la mayor cantidad de árboles se encuentran en las categorías II y III. En general, la forma de la distribución diamétrica del "arrayan" indica un problema de falta de regeneración natural y de haber estado sometida a fuerte presión por la tala.

Regeneración natural.- La regeneración natural en las lomas de Atiquipa alcanza el $33.12 \%$, de los 54 individuos el $66.67 \%$ fueron brinzales y el $33.33 \%$ plántulas (Tabla 3).

La germinación y establecimiento natural a partir de semillas parece ser escasa y las tasas de supervivencia de los brinzales es baja, esto debido posiblemente a que esta especie posee semillas recalcitrantes, esto quiere decir que son sensibles a la deshidratación y tienden a una rápida pérdida de viabilidad posterior a la diseminación (com pers. Carmelo Talavera 2013), a la tala (Rundel et al. 1991, Suasnabar et al. 2004), o a la falta de recurso hídrico y la herbívoria (Jiménez et al. Proyecto el Niño, Contrato ICA-CT-2001-10051, datos no publicados). Se sabe que los ecosistemas de lomas son particularmente susceptibles a la herbivoría (Pefaur 1982). Sin embargo, también se ha documentado que los eventos lluviosos de El Niño (ENSO), pueden mejorar el reclutamiento de especies arbóreas en ecosistemas áridos y semiáridos, estos eventos serian ventajosos para la recuperación de estas especies ante la herbivoría (Holmgren et al. 2006).

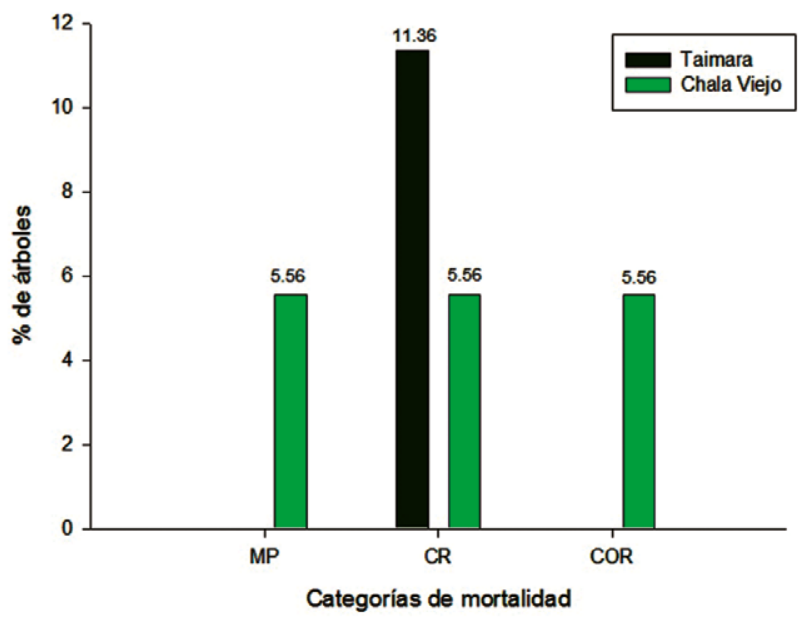

Figura 4.- Número de árboles de Myrcianthes ferreyrae por categoría de mortalidad en cada localidad. Dónde: MP: muerto en pie, CR: caído de raíz, COR: cortado
Mortalidad.- El 7.34 \% del total de árboles, presentaron alguna categoría de mortalidad, siendo la más común la tala (COR) que representa el 75\%; el 25\% restante se distribuye entre árboles caídos de raíz (CR) y muertos en pie (MP) con $12.5 \%$ en ambos casos. El mayor porcentaje de mortalidad (16.67\%) se registró en Chala Viejo en las tres categorías; en Taimara tan solo se registraron árboles caídos de raíz (Fig. 4). No se reportó mortalidad en las parcelas evaluadas en Atiquipa (Fig. 5). Fuera de las parcelas de evaluación, en las localidades de Taimara y Chala Viejo se registraron tocones y árboles caídos, esto constituye una evidencia de la presión antrópica que sufrió esta especie (Rundel 1991). Vizcarra (2004) señala un aumento de la presión de extracción y uso de los bosques por parte de los pobladores y comuneros de Chala y Yauca, que son los que se encuentran más cerca de las Lomas de Chala Viejo. Se sabe que la cercanía de los bosques a las zonas urbanas es un factor determinante para que el riesgo de aprovechamiento del mismo y posible cambio del uso de suelo se incremente.

La presencia de árboles de $M$. ferreyrae muertos en pie, también fue reportado por Suasnabar et al. (2004) y CIZA ORNERN SENAMHI (1989) esto nos hace pensar que podría deberse a un efecto multiestrés (Manion 1991 en Navarro et al. 2008), ya que esta hipótesis enfatiza la interacción de factores bióticos, abióticos y las diferentes formas de estrés antrópico, que juntas podrían conllevar a la muerte de los árboles (Klap et al. 2000 en

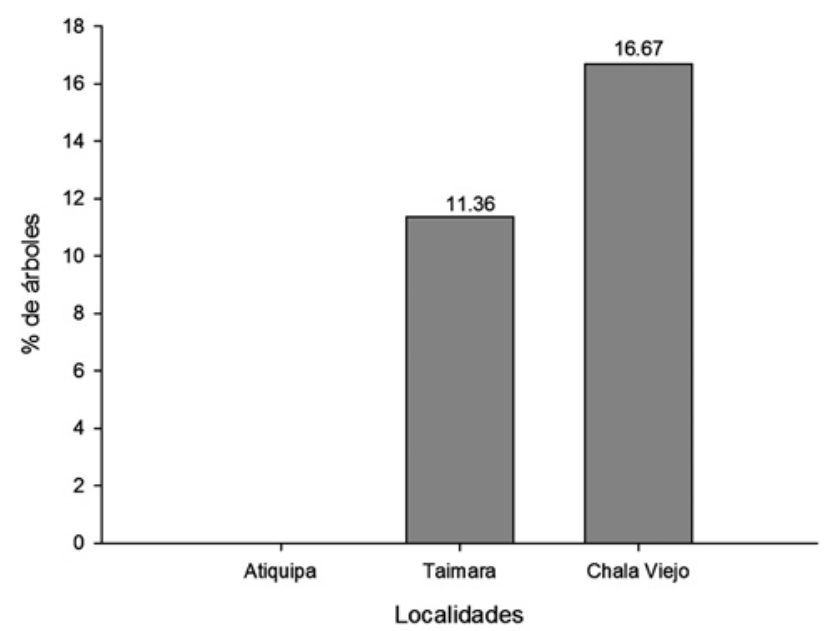

Figura 5.- Porcentaje de árboles de Myrcianthes ferreyrae en cada localidad por categoría de mortalidad 
Navarro et al. 2008). A pesar de que sólo el 5.56\% de los arboles evaluados presentan esta característica, nos parece interesante en un futuro poder describir los agentes causantes de este proceso ecológico.

Degradación de bosque.- Todas las localidades presentaron evidencia obvia de impactos negativos, principalmente por ganadería, ya que todas las localidades evidenciaron signos de pastoreo. La introducción de nuevas especies de animales con el establecimiento del virreinato, como caballos y hatos caprinos, produjo un sobrepastoreo que hizo cada vez más difícil la recuperación anual de las lomas hasta el día de hoy (CIZA ORNER SENAMHI 1989). La herbívora produce principalmente la pérdida de área foliar, y consecuentemente la intercepción de luz por la planta (Reigosa et al. 2004), pudiendo ser esta la principal causa de mortalidad de esta especie, como lo reporta Flores (1997) para los árboles de las lomas de Mejía. Con base a estas premisas consideramos al pastoreo como una de las principales causas de la baja regeneración natural de la especie.

Estado de conservación y amenazas.- Myrcianthes ferreyrae es una especie cuya población ha quedado reducida a unos pocos cientos de individuos, debido principalmente a la sobre explotación del bosque y a la falta de protección oficial en los ambientes donde crece, se protege menos del 15\% de su población en el Área de Conservación Privada Lomas de Atiquipa. Se considera que el área de distribución actual de la especie es el remanente que quedó de su distribución pasada (Rundel et al. 1991), es probable que queden tan solo tres parches relictos de bosque (Atiquipa, Taymara y Chala Viejo), debido a que no existen reportes actuales de las otras localidades donde se distribuye la especie.

Uno de los principales factores que afecta su reproducción y perpetuación sería el aislamiento de los parches que no permitiría su viabilidad en el futuro. Este aislamiento podría estar afectando los procesos de polinización, ya que se conoce de la fuerte asociación entre las Myrtaceae y los Colletidae (himenópteros) (Michener 1976 en Lughadha \& Proenca 1996). Por ello, se puede considerar que la polinización podría ser un proceso importante para las poblaciones que se encuentran cercanas, que serian Huayahuani, Conchara y Avalos y tal vez hasta Atiquipa-Taimara; mientras que no habría intercambio genético con Chala Viejo debido a la distancia que la separa de las otras áreas $(9.54 \mathrm{~km}$ de Taimara).

A lo anterior se suma que la dispersión de $M$. ferreyrae se da por zoocoria (animales) y baricoria (viento), a pesar de ello, por su condición recalcitrante, su establecimiento estaría condicionado también por la humedad del sustrato, condiciones que se asociarían al bajo porcentaje de regeneración natural que se registra históricamente. Debido a los problemas mencionados anteriormente, $M$. ferreyrae podría estar sometida a deriva genética y endogamia, fenómenos que se producen más rápidamente en poblaciones pequeñas como esta (Smith \& Smith 2001).
Finalmente, se observa la falta de estrategias locales y nacionales como la carencia de planes para su conservación y los pocos estudios que se han realizado enfocados a $M$. ferreyrae.

\section{Literatura citada}

Andrewartha HG., \& L.C. Birch. 1984. The Ecological Web. University of Chicago Press, Chicago.

Allen, C. D., Macalady, A. K., Chenchouni, H., Bachelet, D., McDowell, N., Vennetier, et al. 2010. A global overview of drought and heat-induced tree mortality reveals emerging climate change risks for forests. Forest ecology and management, 259(4), 660-684. https://doi. org/10.1016/j.foreco.2009.09.001

Álvarez J. 2014. Fenología de Myrcianthes ferreyrae (Mc. Vaugnt, 1958) en función de la temperatura y humedad relativa en las lomas de Atiquipa (septiembre 2012-agosto 2013), Caravelí-Arequipa. Tesis para optar el grado académico de biólogo, Universidad Nacional San Agustín de Arequipa, Arequipa, Perú.

Brack A. 1986. Las Ecorregiones del Perú. Boletín de Lima. 8(44):57-70

Brako L., \& J. Zarucchi. 1993. Catalogue of the Flowering Plants and Gymnosperms in Peru. Mongr. Missouri Bot. Gard.45.

Brack A., \& C. Mendiola. 2012. Ecología del Perú. Editorial Bruño. Lima.

CIZA-CONCYTEC-ONERN-SENAMHI. 1989. Aprovechamiento de nieblas Costeras en las zonas áridas de la costa. Lomas de Atiquipa (Provincia de Caraveli). Dpto. de Arequipa.

Canziani J. 1997. Atiquipa: un caso prehispánico de manejo sustentable en ecología de Lomas. Páginas 503-526. En: E. González, B. Revesz \& A. Tapia (editores.) Perú: El problema agrario en debate. Seminario Permanente e Investigación Agraria VI. Lima-Perú.

Contreras F., C. Leaño, J. Licona., E. Dauber., L. Gunnar., N. Hager \& C. Caba. 1999. Guía para la Instalación y Evaluación de Parcelas Permanentes de Muestreo (PPMs) Santa Cruz de la Sierra, Bolivia. (Ed). BOLFLOR. http://pdf. usaid.gov/pdf_docs/Pnacg821.pdf

DS N.043-2006. Aprueban la Categorización de especies amenazadas de flora silvestre. Ministerio de Agricultura y Riego MINAGRI.13 de julio 2006. El Peruano Normas Legales: 323527-323539.

Ferreyra R.1983.Los tipos de vegetación de la costa peruana. Anales del Jardín Botánico de Madrid 40(1):241-256.

Ferreyra R. 1986.Flora y vegetación del Perú. En: Gran Geografía del Perú, Naturaleza y Hombre. Volumen II: 1-174. Barcelona, Manfer. Juan Mejía Baca Editores.

Galán de Mera A., E. Linares, C. Trujillo \& F. Villasante. 2010. Termoclima y humedad en el sur del Perú. Bioclimatología y bioindicadores en el departamento de Arequipa. Zonas Áridas 14: 7182.

Gonzales F. 2014. Myrcianthes ferreyrae. The IUCN Red List of Threatened Species 2014: e.T60492231A60492909. http://dx.doi.org/10.2305/IUCN.UK.2014-3.RLTS T60492231A60492909.en.

Holmgren M., B.C.Lopez, J.R. Gutierrez \& F.A. Squeo. 2006. Herbivory and plant growth rate determine the success of El Niño Southern Oscillation-driven tree establishment in semiarid South America. Global Change Biology, 12(12): 2263-2271. https://doi.org/10.1111/ j.1365-2486.2006.01261.x 
Lughadha E., \& C. Proenca. 1996. A survey of the reproductive biology of the Myrtoideae (Myrtaceae). Annals of the Missouri Botanical Garden. 83(4): 480-503. Retrieved from http://www.jstor.org/stable/2399990 doi:1

McVaugh R.1958. Flora of Peru. Botanical series Field Musean of Natural History: Volum. XIII, part IV, number 2.Chicago

Melo 0., \& R. Vargas.2003. Evaluación ecológica y silvicultural de los fragmentos de vegetación secundaria, ubicados en áreas de bosque seco tropical en el norte del departamento del Tolima. Universidad del Tolima. Facultad de Ingeniería Forestal. Ibagué, Colombia. 150 p.

Mancilla R. 2010.Respuesta de dos años de edad de Myrcianthes ferreyrae a diferentes tratamientos de riego en las Lomas de Atiquipa. Universidad Nacional San Agustín, Tesis de Biólogo. Arequipa-Perú.

Navarro G., N. De La Barra, D. Rumiz \& W. Ferreira. 2008. Criterios para evaluar el estado actual de conservación y degradación de los bosques de Bolivia. Revista Boliviana de. Ecología y Conservación. Ambiental 22: 01-18.

ONERN (PERÚ. OFICINA NACIONAL DE EVALUACIÓN DE LOS RECURSOS NATURALES). 1976. Mapa ecológico del Perú; guía explicativa. ONERN. Lima. 146pp.

Pefaur J.1982. Dynamics plants communities in the lomas of Souther Peru. Thesis of PhD. Dissertation. University of Kansas.

PERÚ. ONERN-CIZA-SENAMHI. 1989.Inventario y evaluación de los recursos naturales. En Aprovechamiento de nieblas costeras en las zonas áridas de la costa. Lomas de Atiquipa (prov. Caravelí, dpto. De Arequipa). CONCYTEC. Lima.

Rundel P., M. Dillon, B. Palma, H. Mooney, S. Gulmon \& J. Ehleringer. 1991. The phytogeography and ecology of the coastal Atacama and Peruvian deserts. Aliso 13: 1-49. http://dx.doi.org/10.5642/aliso.19911301.02

Rovere A., A. Premoli \& A. Newton. 2002. Estado de conservación del ciprés de las Guaitecas (Pilgerodendron uviferum (Don) Florín) en Argentina. Bosque (Valdivia) 23 (1):11-19.

Reigosa M., N. Pedrol \& A. Sanchez. 2004. La Ecofisiología Vegetal, Una ciencia de síntesis. Editorial International Tomson Editores Spain, Madrid España. 1193 pp.
Ramírez D., L. Balaguer, R. Mancilla, V. Gonzales, D. Coaguila, C. Talavera, L. Villegas, A. Ortega, P. Jimenez \& J. Moreno. 2011. Leaf-trait responses to irrigation of the endemic fog-oasis tree Myrcianthes ferreyrae: can a fog specialist benefit from regular watering? Tree Physiology 00: 1-9. http://dx.doi.org/10.1093/treephys/tpr121

Smith R.L., \& TM. Smith. 2001. Ecología. Cuarta Edición. Pearson Education. S.A. Madrid 664 pp.

Suasnabar L., O. Franco \& J. Borja .2004. Las áreas de conservación privada en el Perú. Un camino para involucrarse en la conservación de la Naturaleza.Pro Naturaleza. $130 \mathrm{pp}$.

Schulz N., Aceituno P. \& Richter M. 2011. Phytogeographic divisions, climate change and plant dieback along the coastal desert of northern Chile. Erdkunde: 169-187.

Vizcarra G. 2004. Estado actual y análisis de la estructura forestal del bosque de las lomas de Atiquipa. Tesis para optar el grado académico de Biólogo. Universidad Nacional San Agustín, Perú.

Agradecimientos:

Nuestro agradecimiento por el apoyo en campo de Rudy Concha y Gabriel Llerena, y los pobladores de la comunidad de Atiquipa. Agradecemos también a Aldo Ortega, Carmelo Talavera y Luis Villegas por sus consejos, apoyo en la logística y bibliografía. Además, a los revisores por sus comentarios útiles, revisión y criticismo.

Rol de los autores:

FNG realizo el diseño de muestreo, tomo y analizo los datos y redacto el manuscrito; FVB reviso y corrigió el manuscrito. FNG y FVB: revisaron y aprobaron el manuscrito.

Conflicto de intereses:

Los autores no incurren en conflictos de intereses.

Fuentes de financiamiento:

Los autores declaran no haber contado con financiamiento de alguna institución.

Aspectos éticos / legales:

El estudio no requirio permisos especificos y no se realizaron colectas. 
